\title{
The RAD51 and DMC1 homoeologous genes of bread wheat: cloning, molecular characterization and expression analysis
}

\author{
Upendra Kumar Devisetty ${ }^{1,2}$, Katie Mayes $^{1}$, Sean Mayes ${ }^{1 *}$
}

\begin{abstract}
Background: Meiotic recombination in eukaryotes requires two homologues of the E. coli RecA proteins: Rad51 and Dmc1. Both proteins play important roles in the binding of single stranded DNA, homology search, strand invasion and strand exchange. Meiotic recombination has been well studied in Arabidopsis, rice, maize and the orthologues of RAD51 and DMC1 have been characterized. However genetic analysis of the RAD51 and DMC1 genes in bread wheat has been hampered due to the absence of complete sequence information and because of the existence of multiple copies of each gene in the hexaploid wheat genome.

Findings: In this study we have identified that TaRAD51 and TaDMC1 homoeologues are located on group 7 and group 5 chromosomes of hexaploid wheat, respectively. Comparative sequence analysis of cDNA derived from the TaRAD51 and TaDMC1 homoeologues revealed limited sequence divergence at both the nucleotide and the amino acid level. Indeed, comparisons between the predicted amino acid sequences of TaRAD51 and TaDMC1 and those of other eukaryotes reveal a high degree of evolutionary conservation. Despite the high degree of sequence conservation at the nucleotide level, genome-specific primers for CDNAs of TaRAD51 and TaDMC1 were developed to evaluate expression patterns of individual homoeologues during meiosis. QRT-PCR analysis showed that expression of the TaRAD51 and TaDMC1 CDNA homoeologues was largely restricted to meiotic tissue, with elevated levels observed during the stages of prophase I when meiotic recombination occurs. All three homoeologues of both strand-exchange proteins (TaRAD51 and TaDMC1) are expressed in wheat.

Conclusions: Bread wheat contains three expressed copies of each of the TaRAD51 and TaDMC1 homoeologues. While differences were detected between the three cDNA homoeologues of TaRAD51 as well as the three homoeologues of TaDMC1, it is unlikely that the predicted amino acid substitutions would have an effect on the protein structure, based on our three-dimensional structure prediction analyses. There are differences in the levels of expression of the three homoeologues of TaRAD51 and TaDMC1 as determined by QRT-PCR and if these differences are reflected at the protein level, bread wheat may be more dependent upon a particular homoeologue to achieve full fertility than all three equally.
\end{abstract}

\section{Background}

Meiosis is central to all sexually reproducing living organisms and is a highly conserved process. During meiosis a single round of DNA replication is followed by two rounds of cell division resulting in the formation of four daughter cells each having half the chromosome number of the parent. Recombination at meiosis is pivotal not only for

\footnotetext{
* Correspondence: sean.mayes@nottingham.ac.uk

1 Department of Plant and Crop sciences, School of Biosciences, Sutton Bonington Campus, University of Nottingham, Loughborough LE12 5RD, UK Full list of author information is available at the end of the article

Fullist of author information is available at the end of the article
}

creating genetic diversity through crossing-over but also has a mechanistic role ensuring physical connections in the form of chiasmata that allow the chromosomes to segregate accurately during the first division of meiosis. Bacterial RecA was the first gene to be cloned from E. coli that was shown to play an important role in recombination $[1,2]$. Meiotic recombination in eukaryotic cells generally requires two homologues of the $E$. coli RecA protein - Rad51 and Dmc1 - that were first identified in Saccharomyces cerevisiae $[3,4]$. The Rad51 protein forms a nucleofilament with ATP-dependant strand-exchange

C 2010 Mayes et al; licensee BioMed Central Ltd. This is an open access article distributed under the terms of the Creative Commons Attribution License (http://creativecommons.org/licenses/by/2.0), which permits unrestricted use, distribution, and reproduction in any medium, provided the original work is properly cited. 
activity, which has been shown to have roles in meiosis, homologous recombination and DSB repair [5]. Dmc1 also forms a nucleofilament in cooperation with Rad51 and is involved in generating hybrid joint molecules specifically in meiosis [3]. The RAD51 gene exists as a single copy in tomato and Arabidopsis, while maize and Physcomitrella patens have two copies each [6-11]. The DMC1 gene sequences have been reported only in a few plant species. While the Arabidopsis genome contains one copy of the $D M C 1$ gene [12], rice has two copies, OsDMC1A and OsDMC1B, which are located on chromosomes 12 and 11, respectively, most likely produced by chromosomal duplication [13].

Common wheat or bread wheat is an allohexaploid composed of three genomes, each consisting of seven chromosomes (AABBDD; $2 n=6 x=42$ ). Information on the genes and gene homoeologues of RAD51 and DMC1 in bread wheat is limited, primarily due to the added complexity of polyploidy. Given that RAD51 and DMC1 gene orthologues are reported to be involved in meiotic recombination in other cereal species and also the model plant Arabidopsis thaliana, we have investigated their orthologues in bread wheat. A previous study on meiotic recombination genes in wheat reported the existence of only one copy of TaDMC1 gene [GenBank accession number EU915561] and two copies of TaRAD51 gene - TaRAD51A1 [GenBank accession number EU915557] and TaRAD51A2 [GenBank accession number EU915558] [14]. We report here for the first time the existence of three homoeologous copies of the TaRAD51 and TaDMC1 genes, their genomic location and expression patterns in inflorescence and vegetative tissues in bread wheat. These observations in the Chinese Spring wheat do not completely agree with the results reported earlier [14] and these apparent differences are discussed in this paper.

\section{Methods}

\section{Plant materials and DNA isolation}

Wild type hexaploid wheat (Triticum aestivum c.v Highbury) and Nullisomic-Tetrasomic (NT) wheat stocks (Triticum aestivum c.v Chinese Spring) were kindly provided by Prof John Snape and Dr Steve Reader at the John Innes Centre (Norwich, UK). The NT stocks, originally developed by Sears [15], were used for chromosomal assignment of the putative homoeologous genes. Lines Nullisomic for genome A chromosomes were tetrasomics either for B or D chromosomes, lines Nullisomic for genome B chromosomes were tetrasomics either for A or D chromosomes and Nullisomic for genome D chromosomes were tetrasomics either for A or B chromosomes.

Seeds of wheat were first germinated on moistened filter papers in Petri dishes incubated at $20^{\circ} \mathrm{C}$ in a growth chamber. When coleoptile length had reached $\sim 1 \mathrm{~cm}$, seedlings were transplanted to $13 \mathrm{~cm}$ pots containing John Innes No. 3 compost and grown under standard glasshouse conditions. Total genomic DNA was extracted from young leaf tissue (2 - 4 weeks post emergence) using the GenElute ${ }^{\mathrm{Tu}}$ Plant Genomic DNA Miniprep kit (Sigma Aldrich) according to the manufacturer's instructions.

\section{RNA extraction and first strand CDNA synthesis}

Total RNA was isolated from the following tissues: root tips (RT, 14 days after germination), mature leaf (L) and immature inflorescence material (I). In addition, meiotically staged anthers were collected for RNA extraction from anther (microspore) tissues. Stages included are: pre-meiosis (PM), leptotene to pachytene (LP), diplotene to anaphase I (DA), telophase I to telophase II (TT), tetrads (T) and immature pollen (IP). Extractions used Trizol reagent (Gibco-BRL) according to the manufacturer's instructions. The isolated RNA was treated with RQ1 RNase-Free DNase (Promega) to remove any residual genomic DNA according to the manufacturers' instructions and first strand cDNA was synthesized using Superscript III Reverse Transcriptase (Invitrogen) using standard methods.

\section{Isolation of full-length CDNA sequences of wheat RAD51 and $D M C 1$ genes}

Wheat $R A D 51$ and $D M C 1$ genes had not been reported at the start of this research, so in order to isolate the wheat RAD51 cDNA sequence, the rice OsRAD51A1 cDNA sequence [Gen Bank accession number ABO8O261.2] was retrieved from the GenBank database (http://www.ncbi. nlm.nih.gov/genbank) and used to design a total of four primer sets to amplify different sized fragments covering the entire rice cDNA sequence, including $3^{\prime}$ and $5^{\prime}$ UTR sequences (Table 1). These were then used in RT-PCR using wheat immature inflorescence cDNA as the template. The RT-PCR products were cloned, sequenced and aligned into contigs to obtain the full-length TaRAD51 cDNA. To isolate the full-length wheat DMC1 cDNA sequences, primers were designed to the partial $D M C 1$ cDNA sequence of wheat [GenBank accession number DQ247845] and $3^{\prime}$ and 5' RACE was performed. To generate TaDMC1 3 ' ends, mRNA was reverse transcribed using Oligo $\left(\mathrm{dT}_{18}\right)$ primer (Table 1$)$. After first strand cDNA synthesis, the 3 ' region of the TaDMC1 cDNA was amplified with DGSP1 and the 3' NotI anchor primer (Table 1) in the first PCR, and then a nested PCR was done with DGSP2 in combination with the NotI anchor primer (Table 1). 5'-RACE to generate TaDMC1 5' ends was carried out using a 5'RACE System for Rapid Amplification of cDNA Ends kit (Invitrogen) following the manufacturer's instructions. The PCR products were obtained with AAP or AUAP primers (provided in the kit) and gene 
Table 1 List of primers used in the present study

\begin{tabular}{|c|c|c|c|c|}
\hline Primer name & Primer sequence $\left(5^{\prime}-3^{\prime}\right)$ & $\mathrm{Tm}^{\circ} \mathrm{C}$ & Size (bp) & Use \\
\hline OsRAD51Exon 1-2-F & CCTCACATCCCGAGCATCTC & 60 & 171 & Cloning of full-length cDNA of wheat RAD51 \\
\hline OsRAD51Exon 1-2-R & TCACATCAACTGCAGCTATTCCAG & & & \\
\hline OsRAD51 Exon 8-9-F & GGTAGTGGCCCAAGTGGATG & 60 & 564 & \\
\hline OsRAD51 Exon 8-9-R & TGGTGGTCCAATATCACATAGGAG & & & \\
\hline OsRAD51 Exon 1-7-F & ATGTCGTCGGCGGCGG & 65 & 889 & \\
\hline OsRAD51 Exon 1-7-R & CTCATCCGCTAATTTCTGAAGGC & & & \\
\hline OsRAD51 Exon 2-9-F & AAGTCGATAAGATAAT TGAAGCA & 60 & 879 & \\
\hline OsRAD51 Exon 2-9-R & CTGAAACCTTGCTTCAGCT & & & \\
\hline Notl-(dT) 18 & AACTGGAAGAATTCGCGGCCGCAGGAA(T) 18 & & & Cloning of full-length cDNA of wheat $D M C 1$ \\
\hline Notl(dT) anchor & AACTGGAAGAATTCGCGG & & & \\
\hline TaDMC1 -DGSP1 & GAAGAGCCTITCAGGCTTCTG & 55 & 700 & \\
\hline TaDMC1-DGSP2 & GGTGAACTTGCAGAGCGCCAG & 55 & 772 & \\
\hline TaDMC1-DGSP3 & CTGGCGCTCTGCAAGTTCACC & 55 & 772 & \\
\hline TaDMC1-DGSP4 & GGAAGACCCAGTTGGCTCAT & 55 & 461 & \\
\hline TaDMC1-DGSP5 & GGTGAACTTGCAGAGCGTCAG & 55 & 207 & \\
\hline TaRAD51 Exon 8-F & CCATGATGGTGGAGACAAGG & 65 & 2500 & $\begin{array}{l}\text { Amplification of intronic regions in } \\
\text { TaRAD51 \&TaDMC1 genes }\end{array}$ \\
\hline TaRAD51 3'UTR-R & TGGTGGTCCAATATCACATAGGAG & & & \\
\hline TaDMC1-5'UTR-F & ATGATCCACATTCCACCCGC & 65 & 2500 & \\
\hline TaDMC1-Exon 6-R & ATGAGCCAACTGGGTCTTCC & & & \\
\hline iTaRAD51-7A\&7D-F & CGGAAGGATTGGTA AAAAAT & 55 & $450 \& 500$ & $\begin{array}{l}\text { TaRAD51 \&TaDMC1 genome-specific primers based on } \\
\text { intronic regions for localization on wheat genome }\end{array}$ \\
\hline iTaRAD51-7A\&7D-R & CACTCAGAAATGACG AAAAAGG & & & \\
\hline iTaRAD51-7B-F & ATGGTGGAGACAA GGTGAG & 63 & 500 & \\
\hline ITaRAD51-7B-R & GTACACTAGCATTAC GGTACAG & & & \\
\hline iTaDMC1-5A-F & AGCCTCCGCCCCACTTCCTTC & 63 & 500 & \\
\hline iTaDMC1-5A-R & ACAAACGCAACACGA GCACACG & & & \\
\hline iTaDMC1-5B-F & AGCCTTGGCCCCACTTCCTC & 63 & 500 & \\
\hline iTaDMC1-5B-R & ACGCGCTGCACGCA CCAAA & & & \\
\hline iTaDMC1-5D-F & СTCCTCTGACGCAG GCGGA & 63 & 389 & \\
\hline iTaDMC1-5D-R & ACGCGCTGCACGC ACCAAA & & & \\
\hline TaRAD51-UTR-F & CCTCACATCCCGAGCATCTC & 63 & 1528 & TaRAD51 cDNA full-length primers including UTR's \\
\hline TaRAD51-UTR-R & CTCCTATGTGATATTGGACCACCA & & & \\
\hline eTaRAD51-7A-F & GGGGATACCTCGTGTATCAGACT & 58 & 328 & $\begin{array}{l}\text { TaRAD51 genome-specific primers based on } \\
\text { exons for QRT-PCR analyses }\end{array}$ \\
\hline eTaRAD51-R & TGGTGGTCCAATATCACATAGGAG & & & \\
\hline eTaRAD51-7B-F & GATACATCGTGTATCGGACA & 58 & 328 & \\
\hline eTaRAD51-R & TGGTGGTCCAATATCACATAGGAG & & & \\
\hline eTaRAD51-7D-F & TGGGGATACATCGTGTATTGGCCT & 65 & 327 & \\
\hline eTaRAD51-R & TGGTGGTCCAATATCACATAGGAG & & & \\
\hline TaDMC1-UTR-F & ATGATCCACATTCCACCCGC & 63 & 1268 & TaDMC1 cDNA full-length primers including UTR's \\
\hline TaDMC1-UTR-R & CACTGCAGAAAAGAAATTGGGCAAC & & & \\
\hline eTaDMC1-5A-F & AGCCTCCGCCCCACTTCCTTC & 65 & 150 & $\begin{array}{l}\text { TaDMC1 genome-specific primers based } \\
\text { on exons QRT-PCR analyses }\end{array}$ \\
\hline eTaDMC1-R & CTTGTCGATGGACTCGAAGCACTC & & & \\
\hline eTaDMC1-5B-F & AGCCTTGGCCCCACTTC CTC & 65 & 150 & \\
\hline eTaDMC1-R & CTTGTCGATGGACTCGAAGCACTC & & & \\
\hline eTaDMC1-5D-F & TTCTCCTCCAGCAGCACGCGAA & 65 & 150 & \\
\hline eTaDMC1-R & CTTGTCGATGGACTCGAAGCACTC & & & \\
\hline TUB-F & TCTTCATGGTGGGCTTCGC & 55 & 475 & QRT-PCR control primers \\
\hline TUB-R & CGCCTCGGGTGAACTCCATCT & & & \\
\hline
\end{tabular}


specific primers (Table 1). The RT-PCR products were cloned, sequenced and aligned into contigs to obtain the full-length TaDMC1 cDNA.

\section{Generation of Genome-specific primers for TaRAD51 and TaDMC1 using intron sequences}

OsRAD51A1 [GenBank accession number ABO8O261] and OsDMC1A [GenBank accession number AB079873] cDNA sequences were used to predict the exon/intron boundaries of TaRAD51 and TaDMC1, respectively. Once the boundaries were identified, exon-anchored primers that were expected to amplify products spanning two or three introns were designed (Table 1). Multiple alignment of sequences obtained from the TaRAD51 and TaDMC1 cDNA exon-anchored primers on Chinese Spring genomic DNA identified three distinct groups of sequences for each gene with respect to the intronic sequences in bread wheat (data not shown). Putative primers that were expected to amplify a particular homoeologue were designed based on the sequence polymorphisms detected and were coupled with a conserved exon-anchored primer in a PCR reaction containing genomic DNA from Chinese Spring and the appropriate nulli-tetrasomic stocks as templates.

\section{Generation of Genome-specific primers for TaRAD51 and TaDMC1 sequences at the exon level}

Highbury spikelet cDNA was amplified using primers that generate full-length copies of the TaRAD51 and TaDMC1 genes. A major amplification band, approximately $1.5 \mathrm{~kb}$ was obtained with both sets of primers. In the absence of differentially sized fragments, genomespecific primers for TaRAD51 and TaDMC1 cDNA homoeologues were designed based on sequence polymorphisms identified at the 3' UTRs for TaRAD51 cDNA homoeologues and at the $5^{\prime}$ UTRs for the $T a D M C 1$ cDNA homoeologues and were paired with a conserved gene primer (Table 1). The genome specificity of the primers was tested using appropriate nulli-tetrasomic stocks as templates.

\section{Comparative amino acid sequence analysis and 3D protein modelling}

Comparative amino acid sequence analysis was undertaken using the ClustalW tool of European Bioinformatics Institute (http://www.ebi.ac.uk/Tools/clustalw2). The amino acid sequences were processed using the Swiss model workspace (version 8.05, 2009) [16]. The computed outputs were then used to predict the $3 \mathrm{D}$ protein structures using Swiss-Pdb Viewer (DeepView; Version 4.0, 2009) [17].

\section{SIFT (Sorting Intolerant From Tolerant) analysis}

The potential impact of amino-acid changes on the final protein structure were assessed with the SIFT program
[18]. SIFT is a program which predicts whether an amino acid substitution in the protein affects its protein function or not. SIFT assumes that important amino acids will be conserved in the protein family and so changes at well-conserved positions tend to be predicted as deleterious. SIFT score ranges from 0 to 1 . The amino acid substitution is predicted deleterious if the score is $\leq 0.05$ and tolerated if the score is $>0.05$. This approach can only give an indication of potential changes to function through mutation.

\section{Phylogenetic analysis}

The alignment of nucleotide sequences and subsequent selection of best-fit molecular evolution model was performed using Molecular Evolutionary Genetics Analysis (MEGA) software (version 5.0) [19]. The construction of a phylogenetic tree was performed under maximum likelihood (ML) using RAxML version 7.2.6 [20] on the CIPRES portal [21] with the following parameters: nucleotide search using the GTR + G model, random seed for initial parsimony inference, rapid bootstrapping with automatic bootstopping function (frequency criterion) followed by the final ML search.

\section{Quantitative Real-Time PCR}

Harvesting and staging of meiotic anthers for QRT-PCR was performed from immature wheat spikes [22]. Harvested material was immediately frozen and stored at $-80^{\circ} \mathrm{C}$. Eight independent QRT-PCR amplifications were made for the following tissues: PM, LP, DA, TT, T, IP, $\mathrm{L}$ and RT. QRT-PCR was performed in triplicates using wheat Tubulin (Table 1) as an internal standard in a total volume of $12 \mu \mathrm{l}$ reaction that contained $6 \mu \mathrm{l}$ of Brilliant $^{\circ} \mathrm{SYBR}^{\circ}$ Green (Qiagen), $50 \mathrm{ng}$ cDNA and $1 \mu \mathrm{l}$ of an equal mix of forward and reverse gene specific primers (Table 1). The reaction conditions for QRT-PCR were as follows: $15 \mathrm{~min}$ at $95^{\circ} \mathrm{C}$ followed by 45 cycles of $30 \mathrm{~s}$ at $95^{\circ} \mathrm{C}, 30 \mathrm{~s}$ at gene specific annealing temperature and $30 \mathrm{~s}$ at $72^{\circ} \mathrm{C}$. Primer sequences and annealing temperatures are given in Table 1.

Q-PCR amplification reactions were performed in the LightCycler ${ }^{\circ} 480$ instrument (Roche Applied Science, $\mathrm{UK})$ using Brilliant ${ }^{\oplus} \mathrm{SYBR}^{\circ}$ Green (Qiagen) according to the manufacturer's instructions.

\section{Accession numbers}

The cDNA sequences of the TaRAD51 and TaDMC1 homoeologues were deposited in GenBank at NCBI [GenBank accession numbers FJ594479, FJ594480 and FJ594481 for TacRAD51-7A, TacRAD51-7B and TacRAD51-7D homoeologues, respectively and FJ594476, FJ594477 and FJ594478 for TacDMC1-5A, TacDMC1-5B and TacDMC1-5D homoeologues, respectively)]. 


\section{Results and Discussion}

Isolation and genome localization of RAD51 and DMC1 gene homoeologous sequences of hexaploid wheat 'Highbury' wheat spikelet cDNA amplifications resulted in the isolation of the full-length RAD51 and DMC1 cDNA sequences from hexaploid wheat (see methods). In order to localize the isolated RAD51 and DMC1 cDNA sequences on hexaploid wheat, genome specific primers were developed in intronic regions and the genome specificity was confirmed using nulli-tetrasomic analysis. The analysis revealed that the TaRAD51 and TaDMC1 sequences were located on group 7 and group 5 chromosomes, respectively, with a copy in each of the A, B and $\mathrm{D}$ genomes (Figure 1). Exon specific genome specific primers were developed based on sequence polymorphisms identified (Figure 2) and the genome specificity was confirmed using group 7 and group 5 nulli-tetrasomics of wheat (Figure 3). Later full-length cDNA sequences of the TaRAD51 and TaDMC1 homoeologues were isolated using these primers and the sequences were submitted to NCBI database. The locations of TaRAD51 homoeologues cloned here contrast with a previous report [14] in bread wheat that TaRAD51 exists as two paralogues TaRAD51A1 and TaRAD51A2 on group 7.

TaRAD51 and TaDMC1 CDNA homoeologues are highly conserved at the amino acid level

Sequence alignment of the TaRAD51 cDNA homoeologues at nucleotide and amino acid level revealed $97 \%$ and 99\% identity respectively. Two of the TaRAD51 cDNA homoeologues (TaRAD51-7A and $-7 B$ ) have a continuous ORF of $1032 \mathrm{bp}$, capable of encoding a protein of 343 amino acids and the TaRAD51-7D ORF has a deletion of three nucleotides at the $\mathrm{N}$-terminal end, thus putatively encoding a predicted protein of 342 amino acids residues. Further analysis of the three translated TaRAD51 cDNA homoeologues revealed that this leads to the expected deletion of one amino acid at position 17 corresponding to an 'E' residue (Glutamic acid) and single amino acid substitutions are also expected between the three homoeologues at positions 4 (A, compared with $B \& D), 31$ (A\&B, compared with D) and 115 (A\&D, compared with B) (Figure $4 \mathrm{a})$. SIFT predictions suggested that the amino acid substitutions are not expected to affect protein 3D structure for the three cDNA homoeologues of TaRAD51 (Figure $4 \mathrm{~b})$. We have also found that the previously reported [14] TaRAD51A1 is identical to the TaRAD51-7D homoeologue isolated here and that the reported TaRAD51A2 sequence appears to be a truncated version of the TaRAD51-7A homoeologue albeit with a few amino acid differences at the truncated end (listed in Additional file 1). However the paralogous nature of the reported TaR$A D 51 A 1 / A 2$ [14] was not observed in this study. This supports the idea that there is only one copy of TaRAD51 per haploid wheat genome, at least for the wheat genotype studied here. The predicted 3D homoeologue overlays superimposed onto each other TaRad51 revealed there is a high level of predicted conservation for secondary and tertiary structures (Figure 4c). The only noticeable structural dissimilarity observed between the three cDNA

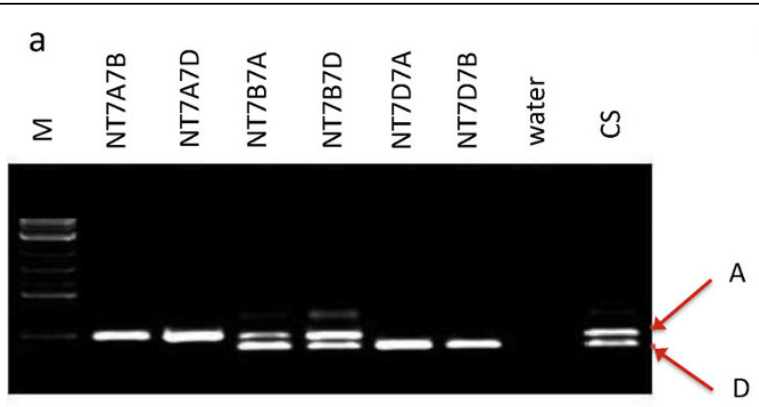

b
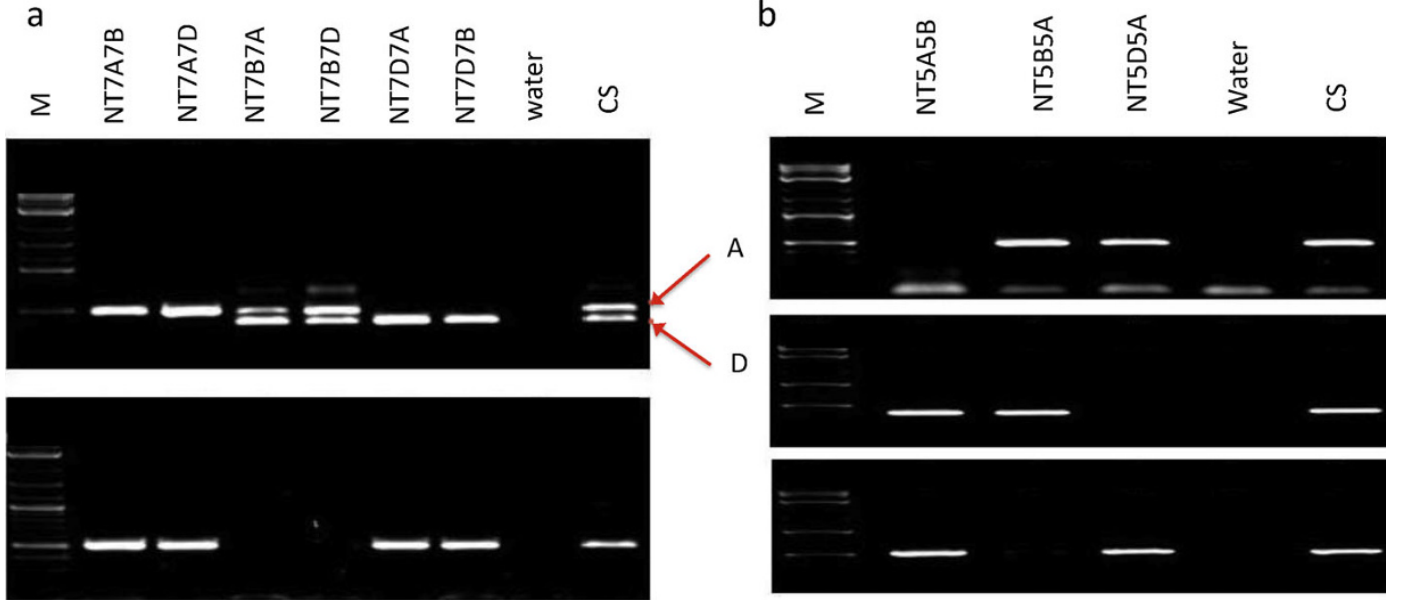

Figure 1 PCR assay for the chromosomal localization of the TaRAD51 and TaDMC1 homoeologous loci, respectively, on group 7 and group 5 of hexaploid wheat. Each primer set designed for TaRAD51 was used to amplify NT7A7B, NT7A7D, NT7B7A, NT7B7D, NT7D7A, NT7D7B, water control and CS. (a) TaRAD51 (A) and (D) genome-specific primer PCR amplifications. The two bands (indicated by red arrows) in Nullisomic 7B and tetrasomic for 7A and 7D lanes can be allocated either to A or D genomes based on PCR amplification on other Nullisomics (Nullisomics 7A and 7D); TaRAD51(B) genome-specific primer PCR amplification and absence of bands in the line nullisomic for 7B and tetrasomic for both 7A and 7D allocates this primer to genome 7B. Each primer set for TaDMC1 was used to amplify NT5A5B, NT5B5A, NT5D5A, water control and CS (b) TaDMC1(A), (B) \& (D) genome-specific primer PCR amplification. Absence of bands in the group 5 Nullisomics allocates the primer to the respective genomes; $M=2-l o g$ ladder (NEB). Only group 5 and group 7 of Nulli-tetrasomics are shown. 


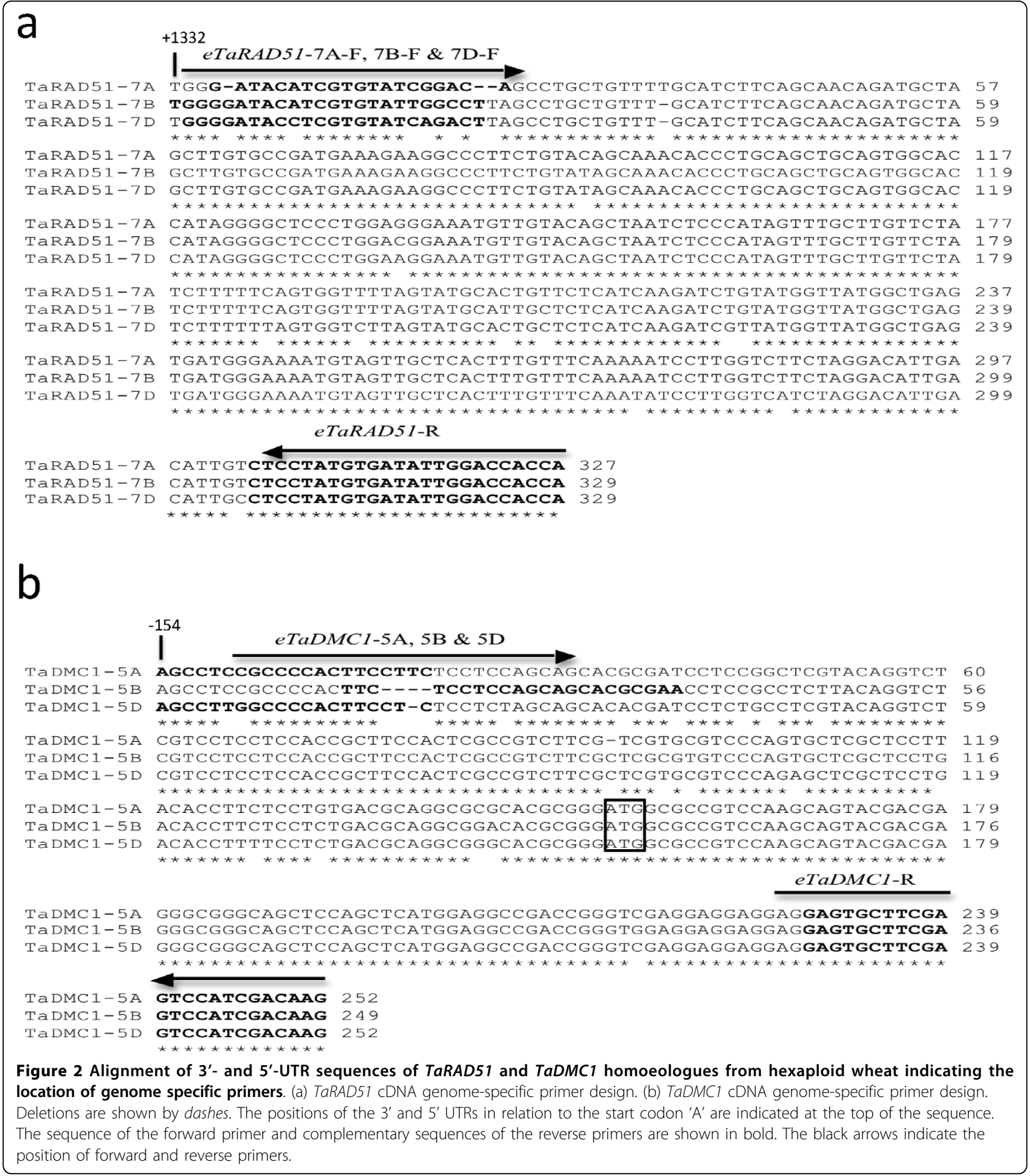

homoeologues is in peptide loops seen between $\alpha$-helix 13 and $\alpha$-helix 14 (indicated by the white arrow in Figure 4c)

Conceptual translation and subsequent sequence alignment of TaDMC1 showed 97\% nucleotide sequence identity and $98 \%$ identity at the amino acid level between the three translated homoeologous copies. All
cDNA homoeologues of the TaDMC1 have a continuous ORF of 1035 bp which encodes a predicted protein of 344 amino acids. Further analysis of the three translated TaDMC1 cDNA homoeologues revealed that the only differences identified between the three cDNA homoeologues were due to nucleotide differences 


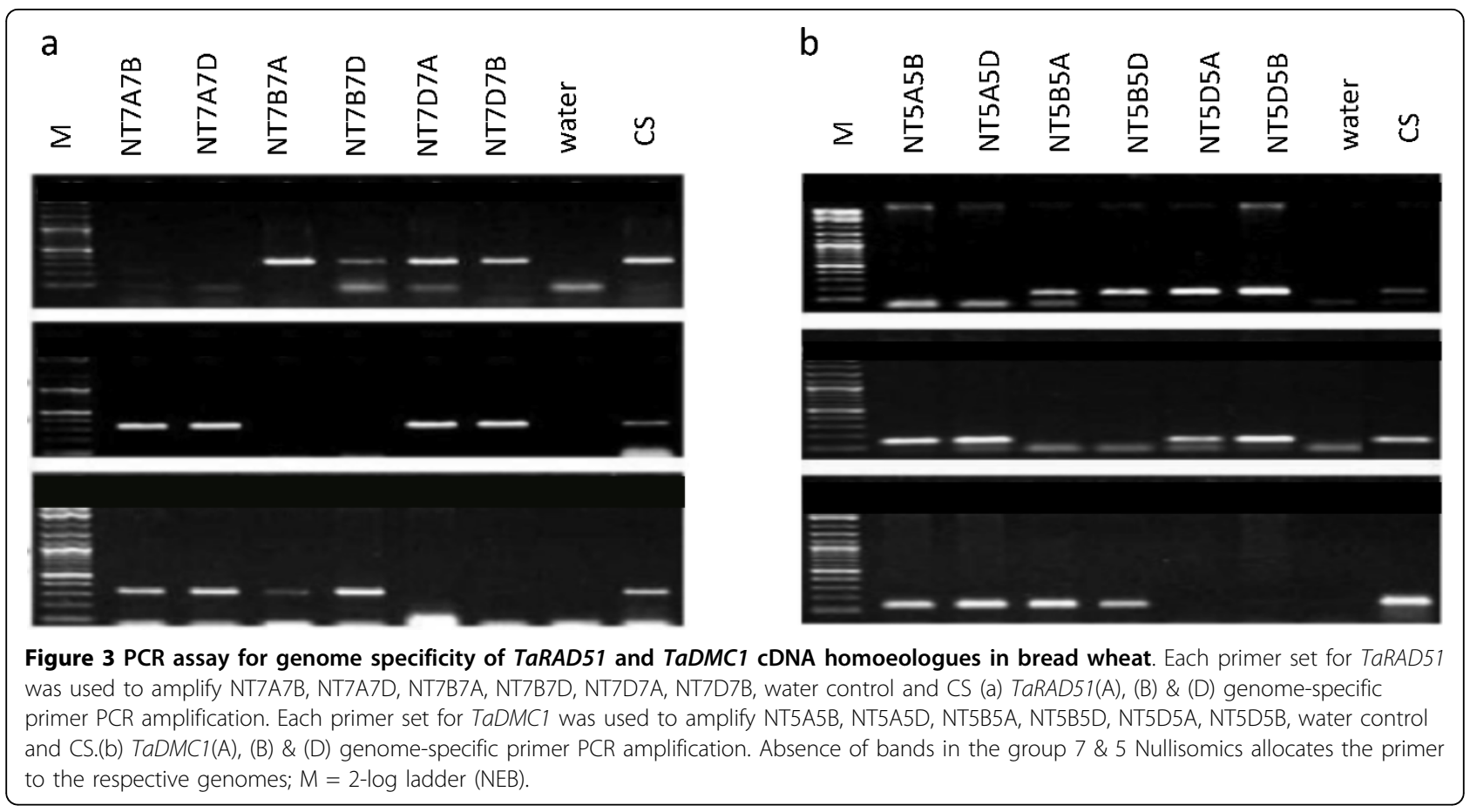

\section{a}

TaRAD51-7A MSSAAAHQKAAAAAPVEEEEAGEHGPFPIE HLQASGIAAVDVKKLKDAGLCTVESVAYSP 60
TaRAD51-7B MSSSAAHQKAAAAAPVEEEAGEHGPFPIEHLQASGIAAVDVKKLKDAGLCTVESVAYSP 60 TARAD51-7D MSSSAAHOKAAAAAPV EEEAGEHGPFPIEQLQASGIAAVDVKKLKDAGLCTVESVAYSP 59

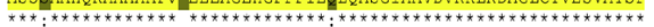

TaRAD51-7A RKDLLQIKGISEAKVDKIIEAASKLVPLGETSATQLHAQRLEIIQVTTGSRELD\#ILEGG 120 TaRAD51-7B RKDLLQIKGISEAKVDKIIEAASKLVPLGFTSATQLHAQRLEIIQVTTGSRELDRILEGG 120 TaRAD51-7D RKDLLQIKGISEAKVDKIIEAASKLVPLGTSATQLHAQRLEIIQVTTGSRELDKILEGG 119

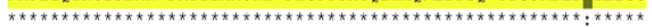

TaRAD51-7A IETGSITELYGEFRSGKTQLCHTLCVTCQLPLDQGGGEGKALYIDAEGTFRPQRLLQIAD 180 TaRAD51-7B IETGSITELYGEFRSGKTQLCHTLCVTCQLPLDQGGGEGKALY IDAEGTFRPQRLLQIAD 180 TARAD51-7D IETGSITELYGEFRSGKTQLCHTLCVTCQLPLDQGGGEGKALYIDAEGTFRPQRLLQIAD 179

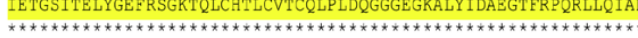

TARAD51-7A RFGLNGADVLENVAYARAYNTDHOSRLLLEAASMMVETRFALMVIDSATALYRTDFSGRG 240 TaRAD51-7B RFGLNGADVLENVAYARAYNTDHQSRLLLEAASMMVETRFALMVIDSATALYRTDFSGRG 240 TARAD51-7D RFGLNGADVIENVAYARAYNT DHQSRLLLEAASMMVETRFALMVIDSATALYRTDFSGRG 239 $* * * * * * * * * * * * * * * * * * * * * * * * * * * * * * * * * * * * * * * * * * * * * * * * * * * * * * * * * * * * * * * *$

TaRAD51-7A ELSARQMHLAKFLRSLQKLADEFGVAVVISNQVVAQVDGGAMFAGPQIKPIGGNIMAHAS $300^{\mathrm{C}}$ TaRAD51-7B ELSARQMHLAKFLRSLQKLADEFGVAVVISNQVVAQVDGGAMFAGPQIKPIGGNIMAHAS 300 TaRAD51-7D ELSARQMHLAKFLRSLQKLADEFGVAVVISNQVVAQVDGGAMFAGPQIKPIGGNIMAHAS 2

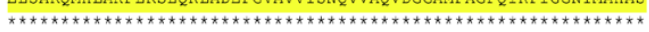

TARAD51-7A TTRLYLRKGRAEERICKVVSSPCLAEAEARFQISPEGVTDVKD 343 TARAD51-7B TTRLYLRKGRAEERICKVVSSPCLAEAEARFQISPEGVTDVKD 343 TaRAD51-7B TTRLYLRKGRAEERICKVVSSPCLAEAEARFQISPEGVTDVKD 343
TaRAD51-7D TTRLYLRKGRAEERICKVVSSPCLAEAEARFQISPEGVTDVKD 342

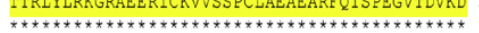

\section{b}

\begin{tabular}{|c|c|c|c|c|}
\hline \multirow{2}{*}{$\begin{array}{l}\text { SNP } \\
\text { Positi } \\
\text { on }\end{array}$} & \multicolumn{3}{|c|}{ TaR AD5I homoeologous genes } & $\begin{array}{c}\text { SIFT } \\
\text { predictio } \\
\text { ns }\end{array}$ \\
\cline { 2 - 5 } & A & B & D & \\
\hline 4 & A & S & S & Tolerated \\
\hline 31 & H & H & Q & Tolerated \\
\hline 115 & K & R & K & Tolerated \\
\hline
\end{tabular}

C

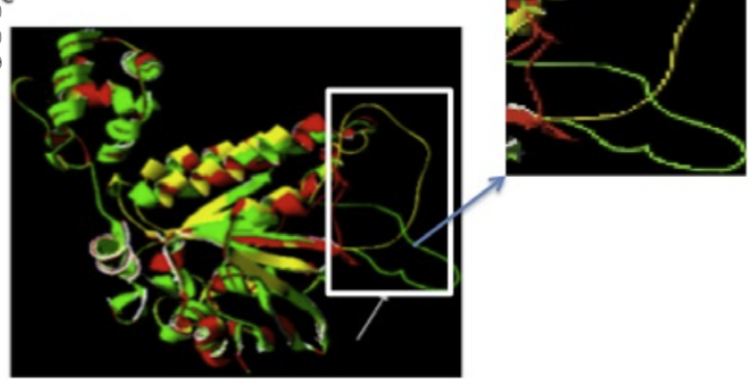

Figure 4 The deduced amino acids alignments of TaRAD51 homoeologous proteins and their 3D models. (a) Multiple alignments of the three TaRAD51 sequences identified in the three bread wheat genomes (,$B$ and D). Conserved amino acids are indicated by black with a yellow background. The amino acid differences between the three cDNA homoeologous proteins are indicated by black with a grey background. (b) SIFT predictions for the amino acid substitutions for the three CDNA homoeologues of TaRAD51. (c) The 3D structure of TaRad51-7A is represented in red, TaRad51-7B in green and TaRad51-7D in yellow. Blue arrow shows the magnified image of the side chains of three TaRad51 homoeologue proteins, which indicates the only structural dissimilarity. 
predicted to produce single amino acid substitutions at positions 114 (A\&D, compared with B), 166 (A\&B compared with D), 214 (A\&B compared with D), 310 (A, compared with $\mathrm{B} \& \mathrm{D})$ and $316(\mathrm{~A} \& \mathrm{~B}$, compared with $\mathrm{D})$; no insertions or deletions were detected (Figure 5a). SIFT predictions for the three translated homoeologues of TaDMC1 suggested that the amino acid substitutions are not expected to affect protein secondary and tertiary structure (Figure 5b). Multiple alignment of the amino acid sequence of the three translated homoeologues of TaDMC1 with the previously reported TaDMC1 cDNA sequence [14] revealed that the reported TaDMC1 is the $\mathrm{D}$ genome homoeologue (data not shown). The predicted 3D overlays for the translated TaDMC1 homoeologues superimposed onto each other revealed very high levels of conservation for secondary and tertiary structures (Figure 5c).

\section{Phylogenetic analysis of TaRAD51 and TaDMC1 CDNA homoeologues reveals evolutionary conservation}

To further analyze the level of conservation and evolutionary relationships of the $\operatorname{Rad} 51$ and Dmc1 predicted proteins across a diverse range of species and to examine the predicted identity of the TaRAD51 and TaDMC1 cDNA homoeologues, previously annotated database entries (listed in Additional file 2) were used to construct a phylogenetic tree. The tree identified three main branches, with Rad51 and Dmc1 members clustered into two separate branches with Rad51B, Rad51C, Rad51D, Xrcc2 and Xrcc3 clustered into a third (Figure 6). This is consistent with the reports that $\operatorname{Rad} 51$ and Dmc1 are paralogues and likely descendants of the ancestral RecA gene [23]. As expected, the three cDNA homoeologues of TaRAD51 and TaDMC1 clustered together and fall into their respective branches. The strongest similarity was found between the nucleotide sequences of wheat and rice $R A D 51$ and $D M C 1$. This provides strong evidence that the cDNA homoeologues isolated in this study are the true orthologues of the rice RAD51 and DMC1 genes.

This study also found that there was only one copy of TaRAD51 per haploid genome of wheat in contrast to two copies of the $R A D 51$ gene reported in rice (OsRAD51A1/A2; rice chromosomes 11 and 12) and maize ( $Z m R A D 51 A 1 / A 2$; maize chromosomes 7 and 8) $[8,13]$. The presence of two RAD51 copies in rice and

\section{a}

TấDMC1-5A MAPSKQYDEGGQLQLMEADRVEEEEECFESIDKLISQGINSG DVKKLQDAGIYTCNGIMM 60 TáDMC1-5D MAPSKQYDEGGQLQLMEADRVEEEEECFESIDKLISQGINSGDVKKLQDAGIYTCNGIMM 60 TâDMC1-5B MAPSKQYDEGGQLQLMEADRVEEEEECFESIDKLISQGINSGDVKKLQDAGIYTCNGIMM 60

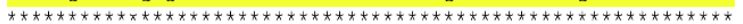

TãDMC1-5A HTRKSLTGIKGLSEAKVDKICEAAEKLISQGFMTGSDLLIKRKSVVRITTGSQALDELLG 120 TâDMC1-5D HTKKSLTGIKGLSEAKVDKICEAAEKLISQGFMTGSDLLIKRKSVVRITTGSQTLDELLG 120 TâDMC1-5B HTKKSLTGIKGLSEAKVDKICEAAEKLLSQGEMTGSDLLIKRKSVVRITTGSQALDELLG 120

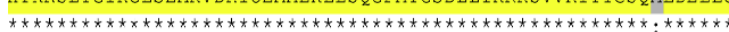

TâDMC1-5A GGIETLCITEAFGEFRSGKTQLAHTLCVSTQLPLHMHGGNGKVAYIDTEGTFRPERIVPI 180 TäDMC1-5D GGIETLCITEAFGEFRSGKTQLAHTLCVSTQLPLHMHGGNGKVAYIDTEGTFRPERIVPI 180 TáDMC1-5B GGIETLCITEAFGEPRSGKTQLAHTLCVSTQLPLHMHGGNGKVAYIGTEGTFRPERIVPI 180

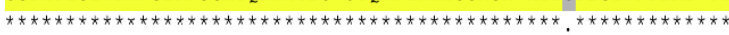

TåDMC1-5A AERFGMDANAVLDNIIYARAYTYEHQYNLLLGLAAAKMAEEPFRLLIVDSVIALERVDFSG 240 TáDMC1-5D AERFGMDANAVIDNIIYARAYTYEHQYNLLLGLAAKMAEEPERLLIVDSVIALLRVDFSG 240 TáDMC1-5B AERFGMDANAVLDNIIYARAYTYEHQYNLLLGLVAKMAEEPERLLIVDSVIALERVDFSG 240 $* * * * * * * * * * * * * * * * * * * * * * * * * * * * * * * * * * *, * * * * * * * * * * * * * * * * * * * * * * * * * * * *$

TáDMC1-5A RG IAERQQKLAQMLSRLTKIAEEFNVAVYITNQVIADPGGGMFITDPKKPAGGHVLAHA 300 TâDMC1-5D RGILAERQQKLAQMLSRLTKIAEEFNVAVYITNQVIADPGGGMFITDPKKPAGGHVLAHA 300 TäDMC1-5B RGILAERQQKLAQMLSRLTKIAEEFNVAVYITNQVIADPGGGMFITDPKKPAGGHVLAHA 300

TádMC1-5A ATIRLMLRKSKGEQRVCKIFDAPNLPEGEAVFQITTGGLMDVKD 344

Tâ.DMC1-5D AT IRLMLRKGKGEQRVCKIFDAPNLPEGEAVFQITTGGLMDVKD 344

TáDMC1-5B ATIRLMLRKGKGEQRICKIFDAPNLPEGEAVFQITTGGLMDVKD 344

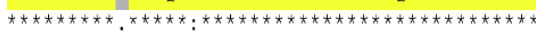

\section{b}

\begin{tabular}{|c|c|c|c|c|}
\hline \multirow{2}{*}{$\begin{array}{l}\text { SNP } \\
\text { Positi } \\
\text { on }\end{array}$} & \multicolumn{3}{|c|}{ TaDMCI homoeologous genes } & \multirow{2}{*}{$\begin{array}{c}\text { SIFT } \\
\text { predictio } \\
\text { ns }\end{array}$} \\
\hline & A & $\mathbf{B}$ & $\mathbf{D}$ & \\
\hline 114 & A & $\mathrm{T}$ & A & Tolerated \\
\hline 167 & D & D & G & Tolerated \\
\hline 214 & A & $\mathrm{A}$ & $\mathrm{v}$ & Tolerated \\
\hline 310 & $\mathbf{s}$ & G & G & Tolerated \\
\hline 316 & v & V & I & Tolerated \\
\hline
\end{tabular}

C

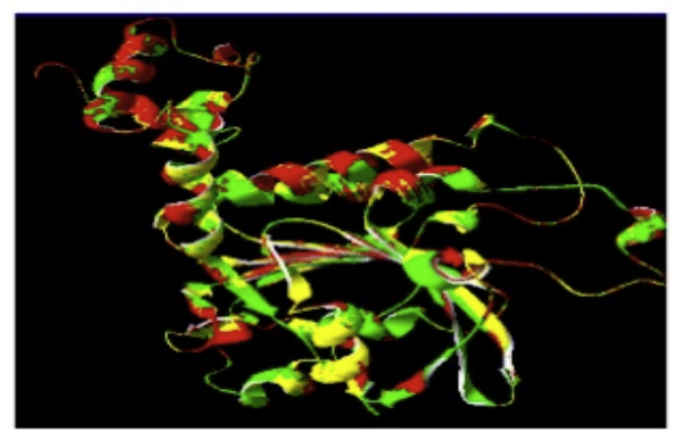

Figure 5 The deduced amino acids alignments of TaDMC1 homoeologous proteins and their 3D models. (a) Multiple alignments of the three TaDMC1 sequences identified in the three bread wheat genomes ( $\mathrm{B}, \mathrm{B}$ and $\mathrm{D})$. Conserved amino acids are indicated by black with a yellow background. The amino acid differences between the three homoeologous proteins are indicated by black with a grey background. (B) SIFT predictions for the amino acid substitutions for the three CDNA homoeologues of TaDMC1. (C) The predicted 3D structure of TaDmc1-7A is represented in red, TaDmc1-7B in green and TaDmc1-7D in yellow. 


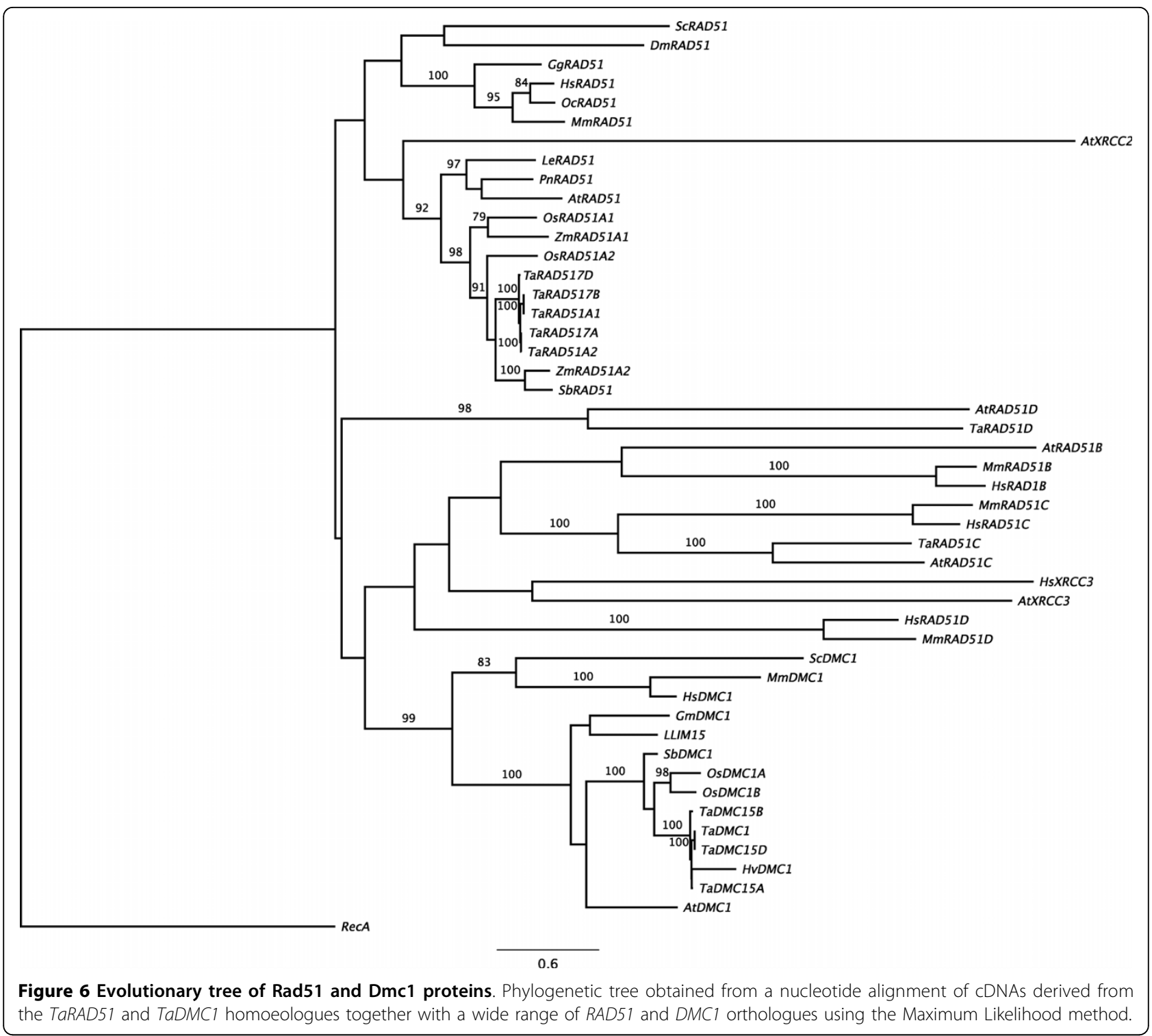

maize and only one copy of RAD51 per genome in wheat may argue for gene loss in the ancestors of wheat. This conclusion is supported by the phylogenetic tree (Figure 6). The genomes of rice and maize contain both products of a predicted earlier gene duplication before divergence of the two species i.e RAD51A1 and $R A D 51 A 2$, while all three of the RAD51 genes in wheat appear to be homologues of the RAD51A2 gene, suggesting that the RAD51A1 lineage has been lost in bread wheat. There are also two versions of the $D M C 1$ gene in rice and maize and only one in the haploid wheat genome. This may have a similar origin, but the greater conservation of DMC1 in these species makes an assessment more difficult and the presence of two copies may simply reflect a local duplication of R11 and R12 in rice and the fact that maize is an ancient tetraploid. In both case, it is worth noting that $D M C 1$ homologues are more highly conserved than the RAD51 homologues, arguing for a higher level of selective pressure preventing mutation or a more recent duplication event.

\section{All homoeologues of TaRAD51 and TaDMC1 genes were highly expressed during prophase I of meiosis}

The QRT-PCR analysis of TaRAD51 cDNA homoeologues indicated that all the three homoeologues were expressed in both vegetative and meiotic tissues (Figure $7 \mathrm{a})$. Comparatively higher expression levels were observed in meiotic stages than vegetative tissues (Figure $7 \mathrm{a}$, stages PM to TT). There is a sharp drop in expression of the TaRAD51 cDNA homoeologue transcripts at the end of Meiosis I but then expression increases again at the Tetrad stage. There was however 


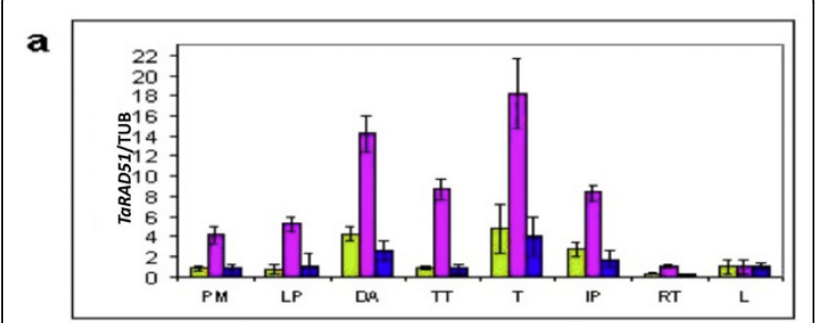

b

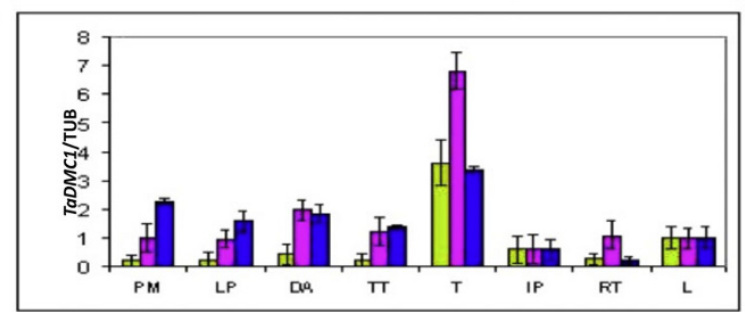

Figure 7 QRT-PCR expression analysis of the TaRAD51 and TaDMC1 homoeologous genes. (a) The green bars represents TaRAD51(A), pink bar represents TaRAD51(B) and blue bar represents TaRAD51(D). (B) The green bars represents TaDMC1(A), pink bar represents TaDMC1(B) and blue bar represents TaDMC1(D).

Abbreviations used: PM, pre-meiotic interphase; LP, leptotenepachytene, DA, diplotene-anaphase I; TT, telophase I-telophase II; T, tetrad; IP, immature pollen; RT, root tips; L, leaves

differences in expression level among the three cDNA homoeologues, particularly during meiotic stages. This may suggest that the individual homoeologues contribute to differing extents in meiotic recombination. The expression level of TaRAD51-7B was significantly higher than TaRAD51-7A and TaRAD51-7D in all meiotic stages suggesting it could be the version with the greatest role in meiosis.

QRT-PCR analysis of TaDMC1 cDNA homoeologues indicated that all three homoeologues were expressed in both vegetative and meiotic tissues (Figure $7 \mathrm{~b}$ ). However higher expression levels were found in meiotic stages (Figure 7b, stages PM to TT) compared to non-meiotic tissues and we observed an increase in expression of all three TaDMC1 cDNA homoeologues during the Tetrad stage. There were differences in the expression levels among all three cDNA homoeologues of TaDMC1 in the observed tissues, which suggests that they could contribute to differing extents in meiotic recombination. Both TaDMC1-5B and TaDMC1-5D homoeologues were highly expressed in all meiotic stages, although not the Tetrad stage, compared to the transcript level of TaDMC1-5A suggesting they could be the versions with the greatest role in meiosis.

Overall, we have reported the cloning and characterisation of the hexaploid wheat versions of the major strandexchange proteins $D M C 1$ and RAD51. Primer tests have been developed which are specific to individual homoeologues and these have been used to characterise the levels of genome-specific expression in a range of tissues through Q-PCR. The same primers could be used to screen mutation and deletion stocks of wheat to detect loss-of-function mutations, allowing in planta characterisation of the effect of these mutations on meiosis.

\section{Additional material}

Additional file 1: Alignment and comparison of the deduced amino acids of TaRAD51 cDNA homoeologues with already reported TaRAD51A1 \&TaRAD51A2 paralogues. Conserved amino acids are indicated by black with a yellow background. The aa similarities between TaRAD51A1 and TaRAD51-7D is indicated by black with green background and aa similarities between TaRAD51A2 and TaRAD51-7A is indicated by black with grey background. Deletions are shown by dashes

Additional file 2: Accession numbers of the nucleotide sequences used in phylogenetic analysis.

\section{Abbreviations}

AA: Amino Acids; DSB: Double Strand Breaks; NT: Nulli-Tetrasomics; RACE: Rapid Amplification of cDNA Ends; CS: Chinese Spring; RT-PCR: Reverse Transcription Polymerase Chain Reaction.

\section{Acknowledgements}

The authors gratefully acknowledge Prof John Snape for Highbury wheat lines, Dr Steve Reader for Nullisomic-Tetrasomic wheat stocks, Dr. Hongying Li for helping with Q-PCR experiments and Dr Jason Able for providing valuable suggestions for meiotic staging of wheat anthers and for useful discussion during the project. UKD was supported by a University of Nottingham Fees PhD Scholarship. The work was partly supported by a New Lecturer grant to SM.

\section{Author details}

${ }^{1}$ Department of Plant and Crop sciences, School of Biosciences, Sutton Bonington Campus, University of Nottingham, Loughborough LE12 5RD, UK. ${ }^{2}$ Department of Plant Biology, 1 Shields Ave, University of California Davis, CA 95616, USA.

\section{Authors' contributions}

UKD conducted the actual research, analyzed the data pertaining to experiments and drafted the manuscript. KM designed the primers for the isolation of TARAD51 and TaDMC1 genes. SM guided the research and proofread this manuscript.

\section{Competing interests}

The authors declare that they have no competing interests.

Received: 25 May 2010 Accepted: 29 September 2010 Published: 29 September 2010

\section{References}

1. Clark AJ, Margulies AD: Isolation and characterization of recombinationdeficient mutants of Escherichia coli K12. Proc Natl Acad Sci USA 1965, 53:451-459.

2. Lusetti, Cox MM: The bacterial RecA protein and the recombinational DNA repair of stalled replication forks. Annu Rev Biochem 2002, 71:71-100.

3. Bishop DK, Park D, Xu L, Kleckner N: DMC1: a meiosis-specific yeast homolog of $E$. coli recA required for recombination, synaptonemal complex formation, and cell cycle progression. Cell 1992, 69:439-456.

4. Shinohara A, Ogawa H, Ogawa T: Rad51 protein involved in repair and recombination in S. cerevisiae is a RecA-like protein. Cell 1992, 69:457-470.

5. Symington LS: Role of RAD52 epistasis group genes in homologous recombination and double-strand break repair. Microbiol Mol Biol Rev 2002, 66:630-670. 
6. Stassen NY, Logsdon JM, Vora GV, Offenberg HH, Palmer JD, Zolan ME: Isolation and characterization of rad51 orthologs from Coprinus cinereus and Lycopersicon esculentum, and phylogenetic analysis of eukaryotic RecA homologs. Curr Genet 1997, 31:144-157.

7. Doutriaux MP, Couteau F, Bergounioux C, White C: Isolation and characterisation of the RAD51 and DMC1 homologs from Arabidopsis thaliana. Mol Gen Genet 1998, 257:283-291.

8. Franklin AE, MCElver J, Sunjevaric I, Rothstein R, Bowen B, Cande WZ: Threedimension microscopy of the RAD51 recombination protein during meiotic prophase. The Plant Cell 1999, 11:809-824.

9. Ayora S, Piruat Jl, Luna R, Reiss B, Russo VEA, Aguilera A, Alonso JC: Characterization of two highly similar Rad51 homologs of Physcomitrella patens. J Mol Biol 2002, 316:35-49.

10. Markmann-Mulisch U, Hadi MZ, Koepchen K, Alonso JC, Russo VEA, Schell J, Reiss B: The organization of Physcomitrella patens RAD51 genes is unique among eukaryotic organisms. Proc Natl Acad Sci USA 2002, 99:2959-2964.

11. Li W, Changbin C, Ullrich MM, Ljudmilla T, Elmon S, Ma H, Reiss R: The Arabidopsis AtRAD51 gene is dispensable for vegetative development but required for meiosis. Proc Natl Acad Sci USA 2004, 101:10596-10601.

12. Klimyuk VI, Jones JD: AtDMC1, the Arabidopsis homologue of the yeast $D M C 1$ gene: characterization, transposon-induced allelic variation and meiosis-assosciated expression. Plant J 1997, 11:1-14.

13. Kathiresan A, Khush GS, Bennett J: Two rice $D M C 1$ genes are differentially expressed during meiosis and during haploid and diploid mitosis. Sex Plant Reprod 2002, 14:257-267.

14. Khoo KHP, Jolly HR, Able JA: The RAD51 gene family in bread wheat is highly conserved across eukaryotes, with RAD51A1 upregulated during early meiosis. Funct Plant Biol 2008, 35:1267-1277.

15. Sears ER: Nullisomic-tetrasomic combinations in hexaploid wheat. In Chromosome manipulation and plant Genetics. Edited by: Riley R, Lewis KR. Oliver 1966:29-45.

16. Bordoli L, Kiefer F, Arnold K, Benkert P, Battey J, Schwede T: Protein structure homology modelling using SWISS-MODEL Workspace. Nat Protoc 2009, 4:1.

17. Kaplan Warren, Littlejohn TG: Swiss-PDB Viewer (Deep View). Brief Bioinform 2001, 2:195-197.

18. Pauline CN, Henikoff S: SIFT: predicting amino acid changes that affect protein function. Nucl Acid Res 2003, 31:3812-3814.

19. Tamura K, Dudley J, Nei M, Kumar S: MEGA4: Molecular evolutionary genetics analysis (MEGA) software version 4.0. Mol Biol and Evol 2007, 24:1596-1599.

20. Stamatakis A, Ludwig T, Meier H: Raxml-iii: A fast program for maximum likelihood-based inference of large phylogenetic trees. Bioinformatics 2005, 21:456-463.

21. Miller MA, Holder MT, Vos R, Midford PE, Liebowitz T, Chan L, Hoover P, Warnow T: The CIPRES Portals.[http://www.phylo.org/sub_sections/ portal].

22. Crismani W, Baumann U, Sutton T, Shirley N, Webster T, Spangenberg G, Langridge $P$, Able J: Microarray expression analysis of meiosis and microsporogenesis in hexaploid bread wheat. BMC Genomics 2006, 7.

23. Lin $\mathrm{Z}$, Kong $\mathrm{H}$, Nei $\mathrm{M}, \mathrm{Ma} \mathrm{H}$ : Origins and evolution of the recA/RAD51 gene family: Evidence for ancient gene duplication and endosymbiotic gene transfer. Proc Natl Acad Sci USA 2006, 103:10328-10333.

doi:10.1186/1756-0500-3-245

Cite this article as: Devisetty et al:: The RAD51 and DMC1

homoeologous genes of bread wheat: cloning, molecular

characterization and expression analysis. BMC Research Notes 2010 3:245.

\section{Submit your next manuscript to BioMed Central and take full advantage of:}

- Convenient online submission

- Thorough peer review

- No space constraints or color figure charges

- Immediate publication on acceptance

- Inclusion in PubMed, CAS, Scopus and Google Scholar

- Research which is freely available for redistribution 